\title{
Membrane-Associated Molecules Guide Limbic and Nonlimbic Thalamocortical Projections
}

\author{
Fanny Mann, ${ }^{1}$ Victoria Zhukareva, ${ }^{3}$ Aurea Pimenta, ${ }^{2}$ Pat Levitt, ${ }^{2}$ and Jürgen Bolz ${ }^{1}$ \\ 1/nstitut National de la Santé et de la Recherche Médicale, Unité 371, Cerveau et Vision, 69500 Bron, France, \\ 2 Department of Neurobiology, University Pittsburgh School of Medicine, Pittsburgh, Pennsylvania 15261, and \\ ${ }^{3}$ Department of Pathology, University of Pennsylvania School of Medicine, Philadelphia, Pennsylvania 15261
}

\begin{abstract}
Membrane-associated signals expressed in restricted domains of the developing cerebral cortex may mediate axon target recognition during the establishment of thalamocortical projections, which form in a highly precise manner during development. To test this hypothesis, we first analyzed the outgrowth of thalamic explants from limbic and nonlimbic nuclei on membrane substrates prepared from limbic cortex and neocortex. The results show that different thalamic fiber populations are able to discriminate between membrane substrates prepared from target and nontarget cortical regions. A candidate molecule that could mediate selective choice in the thalamocortical system is the limbic system-associated membrane protein (LAMP), which is an early marker of cortical and subcortical limbic regions (Pimenta et al., 1995) that can promote out-
\end{abstract}

growth of limbic axons. Limbic thalamic and cortical axons showed preferences for recombinant LAMP (rLAMP) in a stripe assay. Incubation of cortical membranes with an antibody against LAMP prevented the ability of limbic thalamic fibers to distinguish between membranes from limbic cortex and neocortex. Strikingly, nonlimbic thalamic fibers also responded to LAMP, but in contrast to limbic thalamic fibers, rLAMP inhibited branch formation and acted as a repulsive axonal guidance signal for nonlimbic thalamic axons. The present studies indicate that LAMP fulfills a role as a selective guidance cue in the developing thalamocortical system.

Key words: wiring molecules; LAMP; cortical development; cortical areas; limbic system; thalamocortical projections; target recognition; axonal guidance
The mammalian cerebral cortex is characterized by its organization into anatomically and functionally discrete regions. One of the basic features distinguishing each cortical area is the precise pattern of afferent and efferent projections. For example, thalamocortical connections are organized such that specific thalamic nuclei are interconnected with distinct cortical areas. During development in vivo, thalamocortical projections are established very specifically, with thalamic axons bypassing inappropriate areas before invading their appropriate target region (Wise and Jones, 1978; Crandall and Caviness, 1984; De Carlos and O'Leary, 1992; Erzurumlu and Jhaveri, 1992; Agmon et al., 1995). The molecular mechanisms that control the formation of area-specific thalamocortical projections are not known. Co-culture experiments have shown that axons from lateral thalamic nuclei can innervate cortical explants from any region of the cerebral hemisphere (Molnár and Blakemore, 1991; Yamamoto et al., 1992). It has been concluded from these studies that thalamocortical targeting does not depend on molecular cues intrinsic to the cortex (Molnár and Blakemore, 1991, 1995), although normal projections in vivo are highly selective. In contrast, transplantation experiments provided evidence that there might be specific recognition of the correct cortical target by ingrowing thalamic axons (Barbe and Levitt, 1992, 1995).

If the targeting of thalamic axons to their appropriate cortical region is regulated by positional cues intrinsic to the cortex, then

\footnotetext{
Received April 7, 1998; revised Sept. 8, 1998; accepted Sept. 8, 1998.

This work was supported by the Human Frontiers Science Program, a NATO travel grant, and National Institute of Mental Health Grant MH45507. We thank Naura Chounlamountri for excellent technical assistance.

Correspondence should be addressed to Jürgen Bolz, Universität Jena, Institut für Allgemeine Zoologie, Erbertstrasse 1, 07743 Jena, Germany.

Copyright (C) 1998 Society for Neuroscience $\quad 0270-6474 / 98 / 189409-11 \$ 05.00 / 0$
}

cortical areas must exhibit specific molecular labels before thalamocortical innervation. Such molecular markers might be unique for each region, or there might be a few key molecules, distributed in a graded or overlapping manner, to which thalamic axons respond. For example, an enhancer trap transgenic mouse has been identified in which the reporter gene is specifically expressed in the somatosensory cortex (Cohen-Tannoudji et al., 1994). A $29 \mathrm{kDa}$ protein, latexin, is present in a subset of neurons in the infragranular layer of the lateral cortex across several cortical areas (Arimatsu et al., 1992; Hatanaka et al., 1994), and the transcription factor T-brain-1 exhibits a rostrocaudal gradient in the superficial cortical layers (Bulfone et al., 1995). It is not known, however, whether there is a functional link between these molecules and axonal guidance molecules co-expressed in a region-specific manner.

The limbic system-associated membrane protein (LAMP) is another candidate molecule that may be involved in the regional specification of a subset of thalamocortical projections, which at early developmental stages is selectively expressed in the perirhinal and frontal limbic cortex and medial limbic thalamic nuclei (Levitt, 1984; Horton and Levitt, 1988; Pimenta et al., 1996). LAMP can act homophilicly to promote adhesion and growth of limbic axons (Pimenta et al., 1995; Zhukareva and Levitt, 1995), and antibody perturbation studies show that LAMP can regulate the formation of septohippocampal and intrahippocampal circuits (Keller et al., 1989; Pimenta et al., 1995). In the present study, we have examined the influence of native and recombinant LAMP on the growth and guidance of different thalamic axon populations in vitro. Our results indicate that for limbic thalamic axons, LAMP acts as an attractive guidance signal that also can induce branch formation. In contrast, nonlimbic thalamic fibers, which 


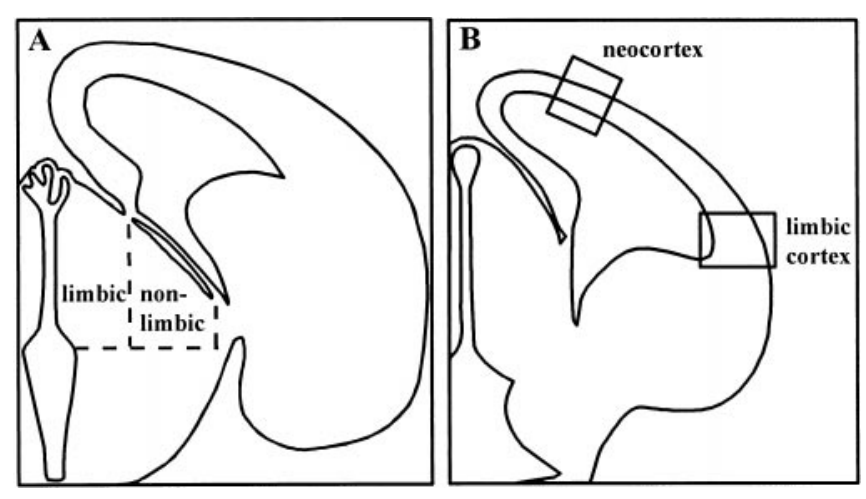

Figure 1. Schematic diagram of coronal sections through E19 $(A)$ and E16 $(B)$ prosencephalon, illustrating the thalamic and cortical regions dissected for in vitro essays. $A$, Dashed lines indicate the medial (limbic) and lateral (nonlimbic) nuclei of the thalamus. $B$, Outlined areas show the regions of the cerebral wall containing cells destined to lateral limbic cortex and parietal neocortex.

normally project to neocortical regions, were deflected and axonal branching was inhibited by LAMP. These results identify a novel role of LAMP on nonlimbic axons. Taken together, our observation suggest that LAMP expressed in limbic cortex acts bifunctionally, attracting limbic thalamic axons and at the same time serving as a repulsive cue to prevent nonlimbic thalamic axons from innervating inappropriate cortical regions.

\section{MATERIALS AND METHODS}

Explant preparation. Sprague Dawley rats at defined stages of pregnancy [day of sperm detection = embryonic day 1 (E1)] were deeply anesthetized with $7 \%$ choral hydrate. Embryos were obtained by cesarean section and decapitated, and the brains were transferred into Gey's balanced salt solution supplemented with glucose $(6.5 \mathrm{mg} / \mathrm{ml})$. Pieces of thalamic and cortical tissues were cut in $200 \mu \mathrm{m}^{3}$ explants with a McIlwain tissue chopper.

Thalamic explants were obtained from E18-E19 fetuses. At this developmental stage, neurogenesis in the thalamus has just finished, and major pronuclei, from which thalamic nuclei differentiate, are formed (McAllister and Das, 1977; Altman and Bayer, 1979). The medial and lateral parts of the differentiating thalamus were separated (Fig. $1 A$ ). In this study, the medial thalamus is called "limbic thalamus," because it gives rise to the main limbic-associated nuclei such as the anterior group of nuclei, the mediodorsal nucleus, and the medioventral nucleus (Krettek and Price, 1977; Robertson and Kaitz, 1981; Deacon et al., 1983). The medial thalamus also includes the habenular complex, which does not project to or receive fibers from the cortex. In some experiments, the habenula was dissected out from the limbic nuclei of the dorsal thalamus. The lateral region of the developing thalamus contains the principal nuclei that project to sensory and motor areas of the cortex; this region will be referred to as "nonlimbic" thalamus. It includes the ventroposterior group of nuclei, which provide inputs to the somatosensory cortex, as well as dorsolateral and medial geniculate bodies, which project to the visual and auditory cortex, respectively (Wise and Jones, 1978; Paxinos and Watson, 1982).

Cortical cells destined for either lateral limbic cortex or neocortex were isolated from E16 embryos at a time when the cerebral wall is essentially composed of subplate and deep cortical plate neurons (Miller, 1988; Bayer and Altman, 1991). Limbic cortical tissue was obtained from the ventrolateral regions of the cerebral wall, at the border with developing striatum and paleocortex, and neocortical explants were prepared from parietal cortex located dorsally (Fig. $1 B$ ).

Preparation of native cortical membranes. Native membranes from young postnatal animals [day of birth, E23 = postnatal day $0(\mathrm{P} 0)$ ] from P3 to P7 were prepared according to the method of Götz et al. (1992). Lateral limbic cortex and medio-dorsal neocortex were dissected in Gey's balanced salt solution supplemented with glucose. Tissues were homogenized in buffer (10 mM Tris- $\mathrm{HCl}, 1.5 \mathrm{~mm} \mathrm{CaCl} 2,1 \mathrm{~mm}$ spermidine, 25 $\mu \mathrm{g} / \mathrm{ml}$ aprotinin, $25 \mu \mathrm{g} / \mathrm{ml}$ leupeptin, $5 \mu \mathrm{g} / \mathrm{ml}$ pepstatin, and $15 \mu \mathrm{g} / \mathrm{ml}$ 2,3-dehydro-2-desoxy- $N$-acetylneuraminic acid, $\mathrm{pH} 7.4$ ), and the homog- enate was centrifuged for $10 \mathrm{~min}$ at $25,000 \mathrm{rpm}$ in a sucrose step gradient. The interface containing the membranes was collected and washed twice at $14,000 \mathrm{rpm}$ in PBS without $\mathrm{Ca}^{2+}$ and $\mathrm{Mg}^{2+}$, supplemented with protease inhibitors (in $\mu \mathrm{g} / \mathrm{ml}: 25$ aprotinin, 25 leupeptin, 5 pepstatin, and 15 2,3-dehydro-2-desoxy- $N$-acetylneuraminic acid). The final pellet was suspended in PBS, and the protein concentration was measured according to the method of Bradford (1976). In some experiments, membranes were incubated for $1 \mathrm{hr}$ at $4^{\circ} \mathrm{C}$ with a monoclonal anti-LAMP antibody (Levitt, 1984; Zacco et al., 1990) at a concentration of $85 \mu \mathrm{g} / \mathrm{ml}$. The treated membranes were washed extensively before use in the outgrowth assays to eliminate unbound antibodies.

Preparation of Chinese hamster ovary cell membranes. Chinese hamster ovary $(\mathrm{CHO})$ cells transfected with pcDNA3-lamp encoding the fulllength recombinant LAMP (rLAMP) or, as a negative control, with pcDNA3 vector alone (Pimenta et al., 1995) were grown in medium consisting of DMEM/F-12 (1:1) supplemented with $10 \%$ fetal bovine serum. To prepare membranes, live cells were washed with PBS. Cells were disrupted by rapid freezing on dry ice and then scraped and collected in homogenizing buffer. Homogenates were incubated for 2-4 $\mathrm{hr}$ at $4^{\circ} \mathrm{C}$ under agitation, and membranes were purified on a sucrose step gradient as described above. Native $\mathrm{CHO}$ cell membranes did not provide a permissive growth substrate for thalamic and cortical explants. For in vitro assays, postnatal neocortical membranes, which have been shown to promote general thalamic and cortical outgrowth (Götz et al., 1992), were mixed (1:2) with CHO cell membranes before use.

Outgrowth assay. Glass coverslips were coated with $1.5 \mu \mathrm{g}$ of laminin and $0.5 \mu \mathrm{g}$ of poly-L-lysine for $1 \mathrm{hr}$ at $37^{\circ} \mathrm{C}$. The coverslips were then separated, washed with PBS, and incubated for $2 \mathrm{hr}$ at $37^{\circ} \mathrm{C}$ with $100 \mu \mathrm{l}$ of membrane suspension with the protein concentration adjusted to 60 $\mu \mathrm{g} / \mathrm{ml}$. Coated coverslips were placed in Petriperm dishes in $750 \mu \mathrm{l}$ of culture medium consisting of 50\% Eagle's basal medium, 25\% HBSS, and $25 \%$ horse serum, supplemented with $0.1 \mathrm{~mm}$ glutamine, $1 \mathrm{mg} / \mathrm{ml}$ glucose, $4 \mathrm{mg} / \mathrm{ml}$ methylcellulose, $100 \mathrm{U} / \mathrm{ml}$ penicillin, and $100 \mu \mathrm{g} / \mathrm{ml}$ streptomycin. Thalamic and cortical explants were pipetted onto the coverslips and incubated for $15-20 \mathrm{~min}$ at $37^{\circ} \mathrm{C}$ to allow adherence to the substrate before adding $1.25 \mathrm{ml}$ of medium. Cultures were kept $2 \mathrm{~d}$ at $37^{\circ} \mathrm{C}$ in an incubator with a humid atmosphere containing $5 \% \mathrm{CO}_{2}$ and then fixed with $4 \%$ paraformaldehyde and $3 \%$ sucrose.

To analyze axonal outgrowth, cultures were observed under an inverted microscope with a $20 \times$ phase-contrast optic. The general outgrowth was assessed by several measures, as described in our previous studies (Götz et al., 1992; Henke-Fahle et al., 1996). The number of fibers extending from each explant was counted, and the length of the five longest fibers was measured for each explant. Statistical differences between experimental conditions were determined using a two-tailed Student's $t$ test. To estimate branching density, axons were examined at a final magnification of $640 \times$. For each explant analyzed, five individual axons, which exhibited a well defined growth cone and no signs of degeneration, were randomly selected. Axonal length was measured, and the number of side collaterals along the axons was counted. The final branching density for each group was calculated by determining the total number of collaterals observed/sum of total axonal length. Statistical differences were determined with Fisher's permutation test.

Stripe assay. Polycarbonate filters containing alternating stripes of two different membrane species were prepared according to the method described by Walter et al. (1987). To permit subsequent analysis of the cultures in phase-contrast microscopy, membrane stripes were transferred onto a glass coverslip coated with laminin and poly-L-lysine as reported by Wizenmann et al. (1993). A potential preference of outgrowing axons for one membrane substrate was assessed by counting the number of fibers growing on each membrane species for a pair of stripes. Axonal numbers on the two different stripes were compared with a two-tailed Student's $t$ test.

Time-lapse video microscopy. Explants were co-cultured either on glass coverslips coated with laminin and poly-L-lysine alone or, to enhance axonal outgrowth, coated with postnatal neocortical membranes. Timelapse video microscopy was performed between 2 and $4 \mathrm{~d}$ in culture to analyze contacts between different axonal populations growing in opposite directions (contact angle, $180 \pm 30^{\circ}$ ). Video images were taken every 90 or $120 \mathrm{sec}$. Details of the procedure for time-lapse video microscopy have been described by Hübener et al. (1995). For LAMP-blocking experiments, explants were cultured on laminin and poly-L-lysine alone, and anti-LAMP was added to the culture medium at a final concentration of $85 \mu \mathrm{g} / \mathrm{ml}$. 


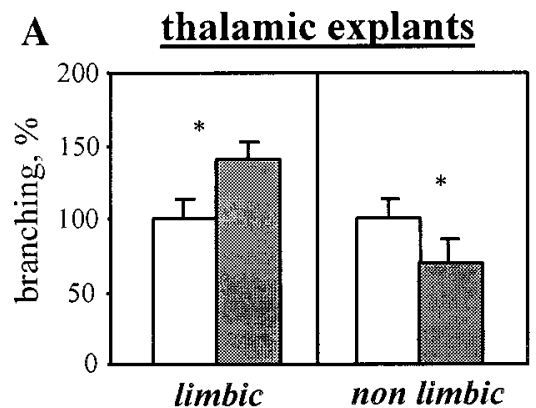

C
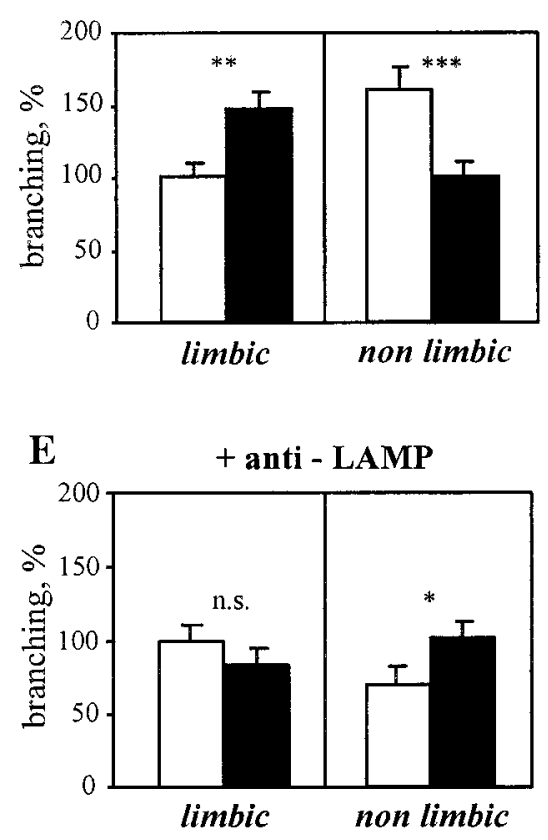

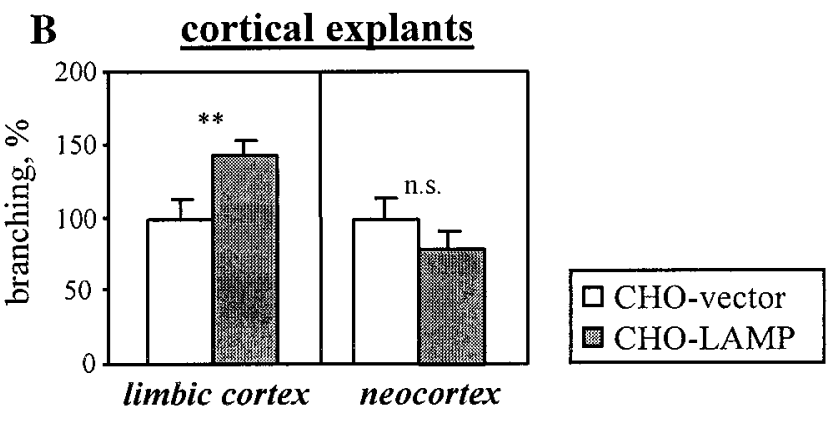

D
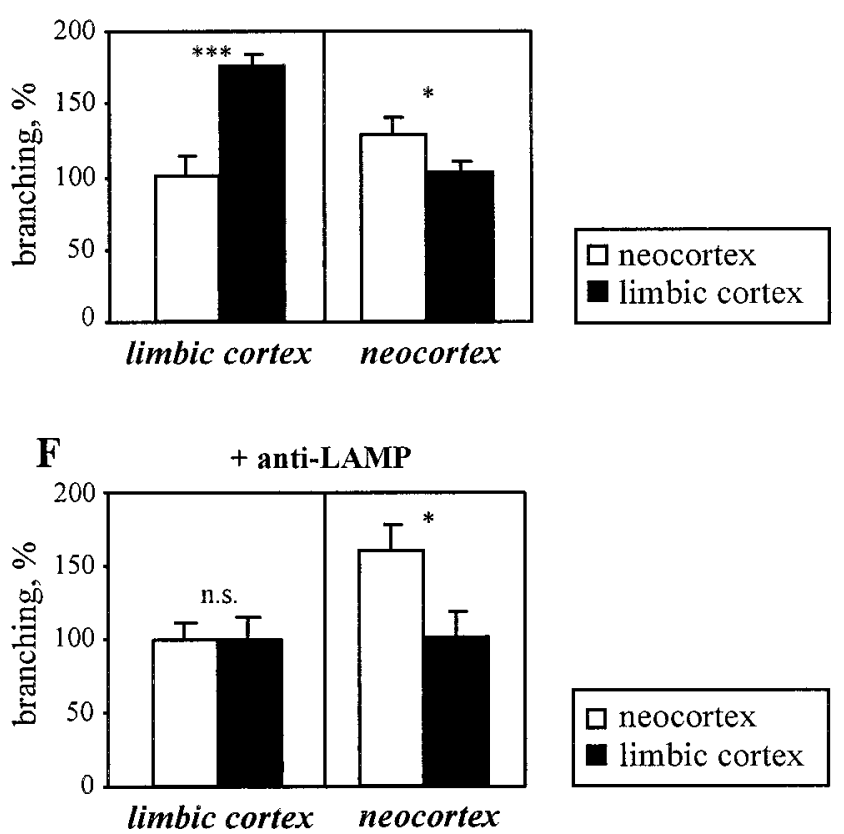

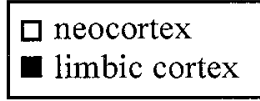

Figure 2. Histograms of branch formation under different experimental conditions. Left, Quantitative analysis for thalamic explants; right, Results for cortical explants. $A, B$, Analysis of growth on rLAMP-expressing or control CHO cell membranes. $C, D$, Branch formation on membranes substrates prepared from postnatal limbic or neocortical areas. $E, F$, Branching on native postnatal cortical membranes preincubated with anti-LAMP. Bars represent the percentage of side branches formed on each substrate; $n$.s., nonsignificant; *significantly different with $p<0.05$; ** significantly different with $p<0.01 ;{ }^{* * *}$ significantly different with $p<0.001$. Statistical analyses are described in Materials and Methods.

\section{RESULTS}

\section{LAMP regulates sprouting and elongation of thalamic and cortical axons}

To explore the functional role of LAMP on cortical and thalamic axons, membrane preparations were extracted from $\mathrm{CHO}$ cell lines stably transfected either with pcDNA3 vector containing an insert encoding full-length rLAMP or, as a control, with the vector alone (Pimenta et al., 1995). In a first set of experiments, the outgrowth of limbic and nonlimbic axons on homogenous substrates of CHO-rLAMP membranes was analyzed and compared with the values obtained on $\mathrm{CHO}$-vector membranes.

Limbic neurons exhibited differential growth behaviors on the two types of substrates, as measured by an increased arborization of limbic fibers when confronted with rLAMP membranes. Quantitative analysis indicated that the branching density of limbic thalamic axons and limbic cortical axons increased 40.7 and $43.2 \%$, respectively, in the presence of rLAMP compared with control conditions [Fig. 2, $A, n=57$ thalamic axons examined (control), $n=57$ (rLAMP); $p<0.05 ; B, n=69$ cortical axons (control), $n=69$ (rLAMP); $p<0.01$ ]. In contrast, neocortical fibers showed no branching preference for either one of the two substrates [Fig. $2 B, n=42$ (control), $n=50$ (rLAMP); NS]. Finally, the branching density of nonlimbic thalamic axons decreased by $30.3 \%$ on the rLAMP substrate compared with control cultures [Fig. $2 A, n=28$ (control), $n=28$ (rLAMP); $p<0.05$ ]. Camera lucida drawings of individual thalamic axons from limbic and nonlimbic explants are presented in Figures 3, $A$ and $B$, and illustrate that LAMP can promote collateral sprouting of limbic axons but, in addition, reduce branch formation of nonlimbic thalamic axons.

Under the same experimental conditions, differences in the general fiber outgrowth of thalamic and cortical explants on CHO-rLAMP membranes versus control membranes were determined. The mean number of fibers per explant and maximal length reached by growing axons are summarized in Table 1. In general, limbic thalamic explants exhibited more outgrowth than nonlimbic thalamic explants. One possible explanation is that neurons from lateral nuclei, which are produced earlier than neurons from medial nuclei (McAllister and Das, 1977), are more differentiated, and their ability to survive in culture may be 
$\mathbf{A}$

membranes of CHO-LAMP cells

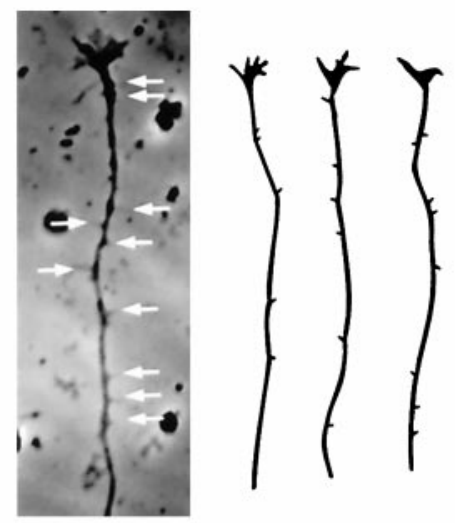

limbic thalamus

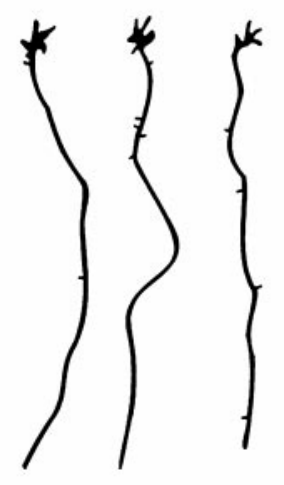

non-limbic thalamus

\section{C membranes of limbic cortex}

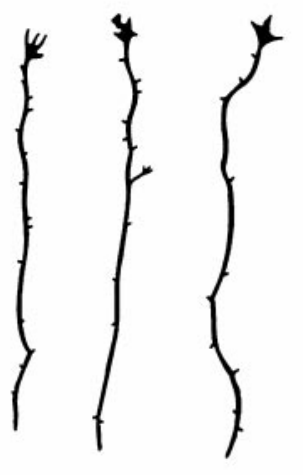

limbic thalamus

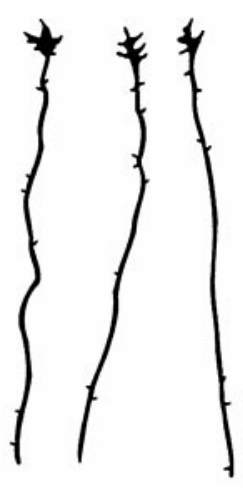

non-limbic thalamus

\section{B membranes of $\mathrm{CHO}$-vector cells}
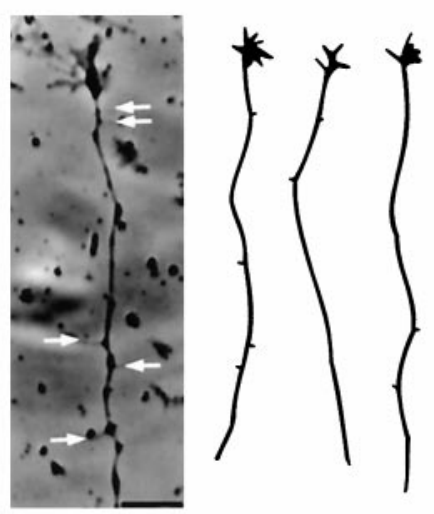

limbic thalamus

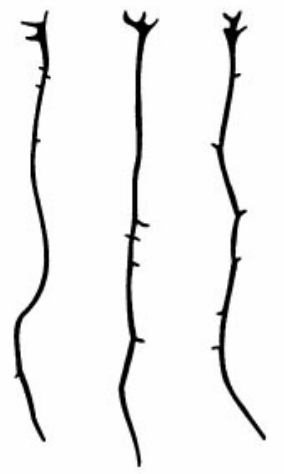

non-limbic thalamus

D membranes of neocortex

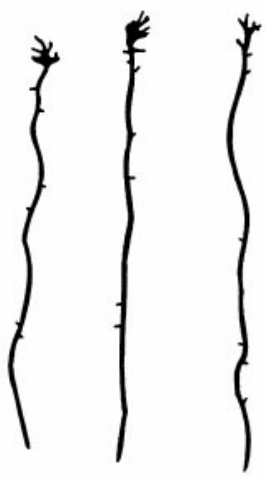

limbic thalamus

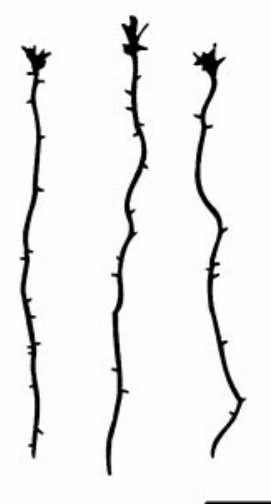

non-limbic thalamus

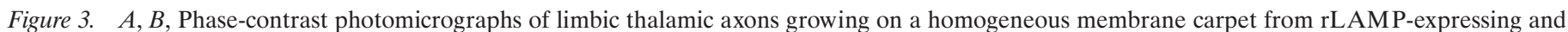

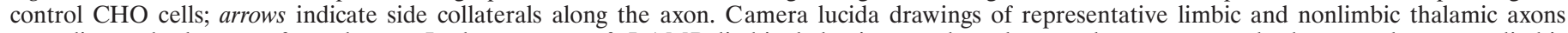

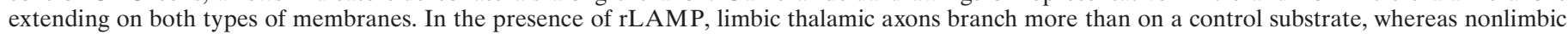

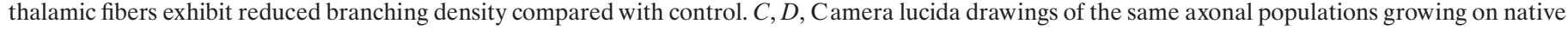

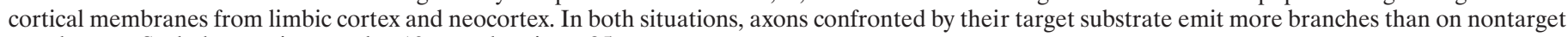
membranes. Scale bars: micrographs, $10 \mu \mathrm{m}$; drawings, $25 \mu \mathrm{m}$.

reduced. Such a relationship between age of thalamic explants and fiber outgrowth in vitro has been reported previously (Bolz et al., 1992). Overall thalamic and cortical explants, independent of their limbic or nonlimbic phenotype, extended similar number of fibers on CHO-rLAMP and control membranes. This indicates that LAMP does not influence general axonal growth, a result similar to that reported previously for septal axons (Keller et al., 1989). However, limbic fibers extending from cortical or thalamic explants grew longer on rLAMP-containing membranes than on control membranes $[n=121$ cortical axons (control), $n=135$ (rLAMP); $p<0.001 ; n=195$ thalamic axons (control), $n=205$ (rLAMP); $p<0.001]$, reflecting a specific growth-promoting effect of substrate-bound LAMP on limbic fibers (Pimenta et al., 1995). The growth pattern of neocortical fibers was unchanged on both substrates, indicating that neocortical fibers are not responsive to LAMP $[n=105$ (control), $n=121$ (rLAMP); NS). Finally, nonlimbic thalamic fibers responded to the presence of
LAMP by exhibiting reduced elongation on CHO-rLAMP membranes compared with control substrate $[n=160$ (control), $n=$ 227 (rLAMP); $p<0.005$ ].

\section{LAMP is a guidance cue for thalamic and cortical fibers}

Our results indicate that, although LAMP promotes branch formation and elongation of limbic fibers, LAMP is not required for outgrowth of limbic fibers. The previous assays, however, were performed independently on CHO-rLAMP or control membranes. It is possible that LAMP could provide guidance cues specifically for limbic fibers when faced with choices. To examine this idea, thalamic and cortical explants were cultured on alternating stripes of CHO-rLAMP membranes and control membranes. Limbic axons were found to grow preferentially on the lanes containing recombinant LAMP. Respectively, 72 and 64\% of limbic thalamic and limbic cortical fibers extended on stripes of 
Table 1. Effects of LAMP on thalamic and cortical outgrowth

\begin{tabular}{|c|c|c|c|c|c|}
\hline \multirow{2}{*}{$\begin{array}{l}\text { Explant } \\
\text { Limbic thalamus }\end{array}$} & \multirow{2}{*}{$\frac{\text { Substrate }}{\text { control }}$} & \multicolumn{2}{|c|}{ Number of fibers (axons/explant) } & \multicolumn{2}{|c|}{ Axonal length $(\mu \mathrm{m})$} \\
\hline & & $28.8 \pm 2.0$ & $n=67$ & $549.7 \pm 11.6$ & $n=195$ \\
\hline Limbic thalamus & LAMP & $29.1 \pm 2.2 \mathrm{NS}$ & $n=50$ & $614.9 \pm 12.5^{*}$ & $n=205$ \\
\hline Nonlimbic thalamus & control & $4.1 \pm 1.0$ & $n=60$ & $348.1 \pm 10.1$ & $n=160$ \\
\hline Nonlimbic thalamus & LAMP & $3.6 \pm 0.8 \mathrm{NS}$ & $n=56$ & $311.2 \pm 7.9 * *$ & $n=227$ \\
\hline Limbic cortex & control & $29.5 \pm 2.2$ & $n=59$ & $502.5 \pm 22.3$ & $n=121$ \\
\hline Limbic cortex & LAMP & $26.4 \pm 2.2 \mathrm{NS}$ & $n=64$ & $638.5 \pm 27.9 *$ & $n=135$ \\
\hline Neocortex & control & $37.7 \pm 1.8$ & $n=66$ & $875.2 \pm 27.6$ & $n=105$ \\
\hline Neocortex & LAMP & $38.9 \pm 1.7 \mathrm{NS}$ & $n=64$ & $834.7 \pm 21.8 \mathrm{NS}$ & $n=121$ \\
\hline
\end{tabular}

Results are mean \pm SEM. For the number of axons per explant, $n=$ number of explants; for axonal length analysis, $n=$ number of fibers examined.

*Significantly different with $p<0.001$.

**Significantly different with $p<0.01$.
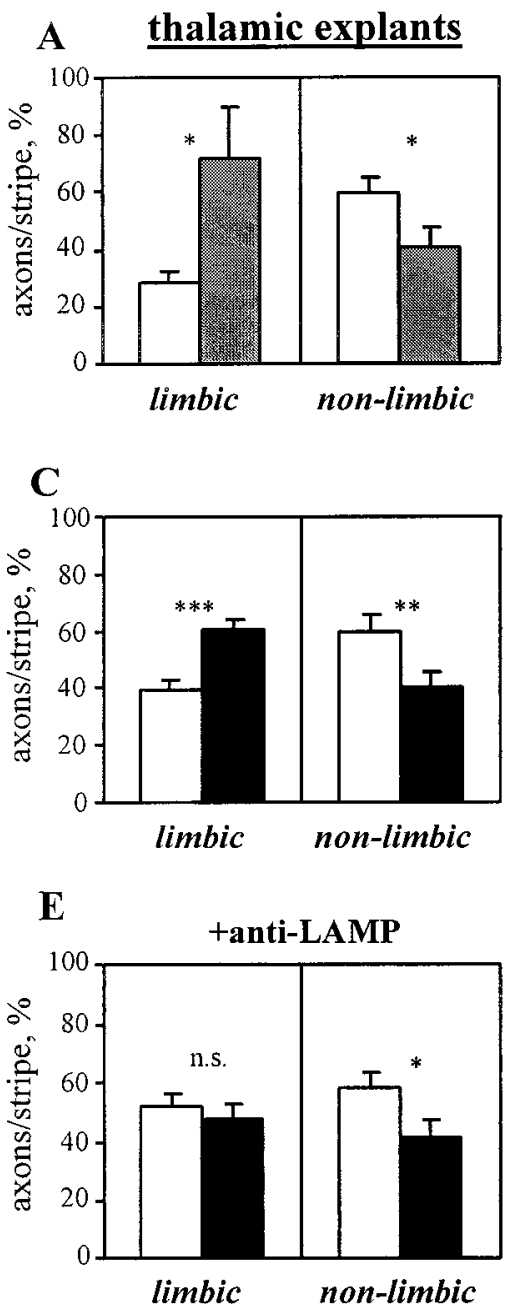

B cortical explants

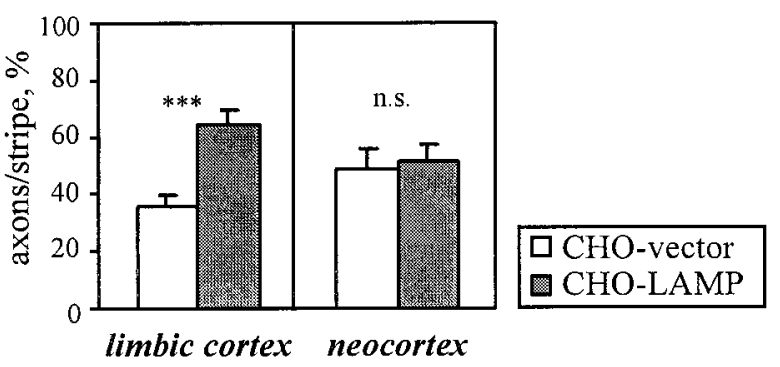

D
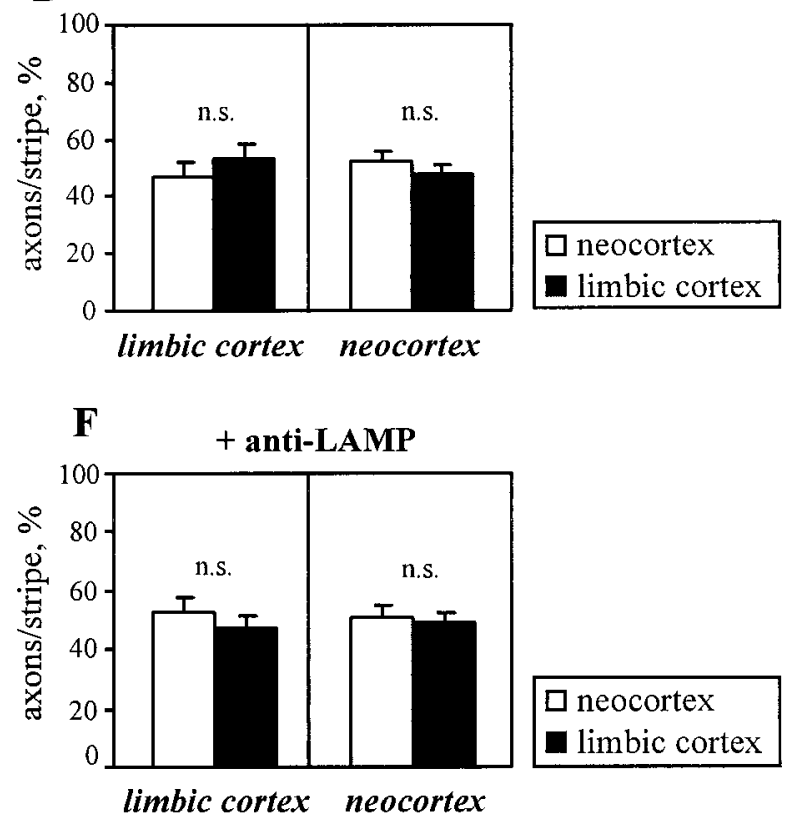

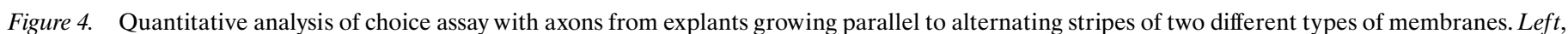

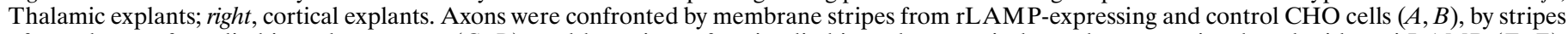

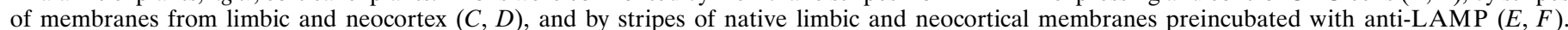

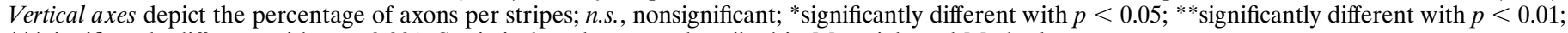
$* * *$ significantly different with $p<0.001$. Statistical analyses are described in Materials and Methods.

CHO-rLAMP membranes (Fig. $4, A, n=40$ pairs of stripes analyzed; $p<0.001 ; B, n=79 ; p<0.001)$. Neocortical axons grew equally on both types of stripes, independent of the presence of LAMP (Fig. $4 B, n=56$; NS). In contrast, $59 \%$ of nonlimbic thalamic fibers extended on control stripes, whereas only $41 \%$ grew on CHO-rLAMP membranes (Fig. $4 A, n=40$; $p<0.05)$. Because the outgrowth assays on homogenous substrates have shown that the presence of rLAMP in the membrane carpet did not influence the number of thalamic and cortical fibers, the differential axonal localization observed here can only 


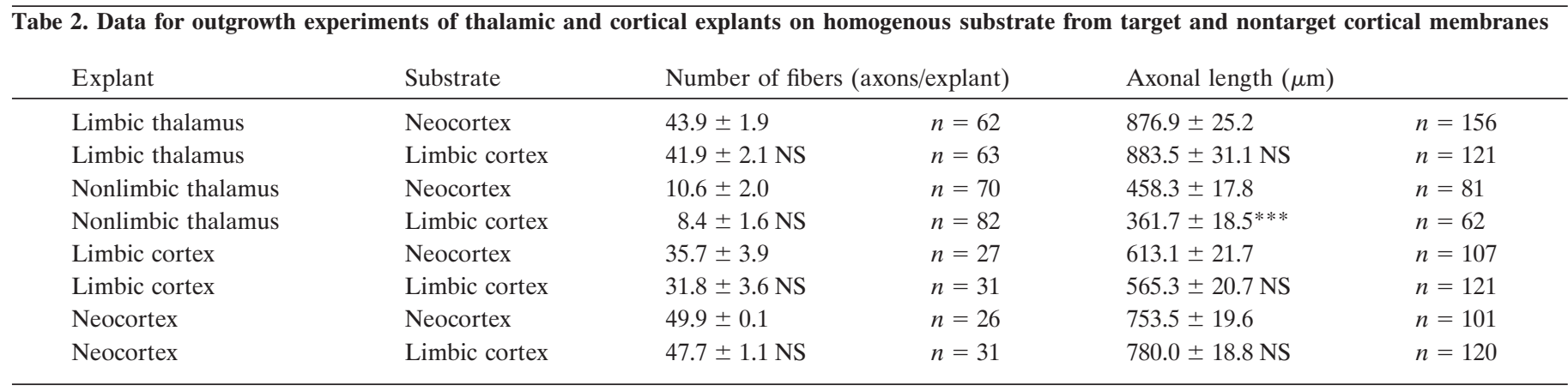

Results are mean \pm SEM. For the number of axons per explant, $n=$ number of explants; for axonal length analysis, $n=$ number of fibers examined.

*Significantly different with $p<0.001$.

be ascribed to a preferential choice for one of the growth substrates. Thus, LAMP acts as an attractive guidance cue for growing axons from limbic-associated cortical and thalamic regions. Moreover, LAMP is able to influence the choice made by nonlimbic thalamic fibers through a repulsive mechanism.

\section{Target recognition by thalamic and cortical axons in vitro}

We used an additional strategy to determine whether limbic and nonlimbic axons have the ability to discriminate between membranes purified from cortical regions expressing differential levels of LAMP. Thalamic and cortical explants were cultured on homogenous substrates composed of postnatal cortical membranes purified from either lateral limbic cortex or parietal neocortex. We analyzed the growth of 819 axons and found that thalamic and cortical fibers exhibited differential branching behaviors on their target and nontarget cortical membranes. As illustrated in Figure 3, $C$ and $D$, on membranes from limbic cortex, limbic thalamic fibers exhibited a higher density of side collaterals than on neocortical membranes. In contrast, sprouting of nonlimbic thalamic fibers was reduced on limbic membranes compared with neocortical membranes. Quantitative analysis of branching showed that limbic thalamic axons and nonlimbic thalamic axons exhibited 45.5 and $59.0 \%$ more side collaterals on their respective target membranes compared with nontarget membranes [Fig. 2C, $n=176$ limbic axons (neocortex), $n=176$ (limbic cortex); $p<0.01 ; n=85$ nonlimbic axons (neocortex), $n=87$ (limbic cortex); $p<0.01$ ]. Likewise, the branching density of limbic cortical fibers and neocortical axons increased by 73.7 and $27.2 \%$ when confronted with their appropriate cortical membranes [Fig. $2 D, n=55$ limbic cortical axons (neocortex), $n=60$ (limbic cortex); $p<0.001 ; n=90$ neocortical axons (neocortex), $n=90$ (limbic cortex); $p<0.05$ ]. These results suggest that membrane-bound signals expressed in defined cortical regions can influence arborization of appropriate cortical and subcortical fibers. Thalamic and cortical cells, harvested during fetal development, are able to respond in vitro to branching signals in a very specific way that reflects their in vivo branching behavior.

We also analyzed axonal length and counted the number of axons extending from cortical and thalamic explants (Table 2). With the exception of nonlimbic thalamic explants, whose fibers extended on average $100 \mu \mathrm{m}$ longer on their target membranes [ $n=81$ (neocortex), $n=62$ (limbic cortex); $p<0.001]$, the overall length of axons from each type of explant was equivalent on the different substrates, indicating that limbic and nonlimbic cortical membranes are similar in supporting the growth of thalamic and cortical fibers.
The stripe assay was used once again to determine the selective ability of limbic and nonlimbic thalamic axons, when confronted with a choice between stripes of limbic and neocortical membranes, to preferentially elongate in the lanes containing their target membranes. Quantitative analysis of axonal distribution revealed that $60 \%$ of limbic thalamic axons grew on limbic cortical stripes, whereas $40 \%$ grew on neocortical lanes (Fig. $4 C, n=$ 106 pairs of stripes; $p<0.001)$. Likewise, $60 \%$ of nonlimbic thalamic fibers extended on neocortical membrane, and $40 \%$ extended on limbic cortical membrane (Fig. $4 C, n=51 ; p<0.01$ ). A subpopulation of thalamic axons thus are able to discriminate membranes from different cortical regions and are guided on their appropriate substrate. In cultures of cortical explants, we failed to observe a preference of growing fibers for any type of substrate. Both limbic and neocortical axons were equally distributed on stripes of target and nontarget cortical membranes (Fig. $4 D, n=$ 52; NS; $n=106$; NS).

In the experiments described above, we analyzed the outgrowth of limbic thalamic explants prepared from the medial thalamus, including the habenular nuclei, which do not project to the cerebral cortex in vivo. Because the habenula could contribute to the observed differential branching and outgrowth on cortical membranes, in control experiments we examined independently the growth of neurons dissected from either limbic dorsal thalamus or habenular complex (Fig. 5A). Overall, habenular axons exhibited only $\sim 20 \%$ as many collaterals as axons from limbic dorsal thalamus (Fig. $5 B$ ). The branching behavior of habenular fibers was unchanged on membranes from limbic and neocortical regions [Fig. $5 B, n=70$ axons (neocortex), $n=48$ (limbic cortex); NS]. Moreover, habenular fibers showed no preference for any type of cortical membranes in the stripe assay (Fig. $5 C, n=24$ pairs of stripes; NS). In the same test situation, however, we found an enhancement of axonal branching and a preferential growth on limbic cortical membranes for neurons of limbic dorsal thalamus [Fig. 5, $B, n=26$ axons (neocortex), $n=26$ (limbic cortex); $p<0.05 ; C, n=7$ pair of stripes; $p<0.05$ ).

\section{LAMP inhibition abolishes target recognition by limbic fibers}

Our results indicate that molecular components expressed in the developing cortex regulate sprouting and trajectory of appropriate thalamic axons. To determine whether LAMP controls target recognition by limbic axons on native membranes, the assays described above were repeated after preincubating membranes with specific antibodies directed against LAMP. The blocking of LAMP activity affected the branching of limbic thalamic and limbic cortical axons, resulting in an absence of preferential 


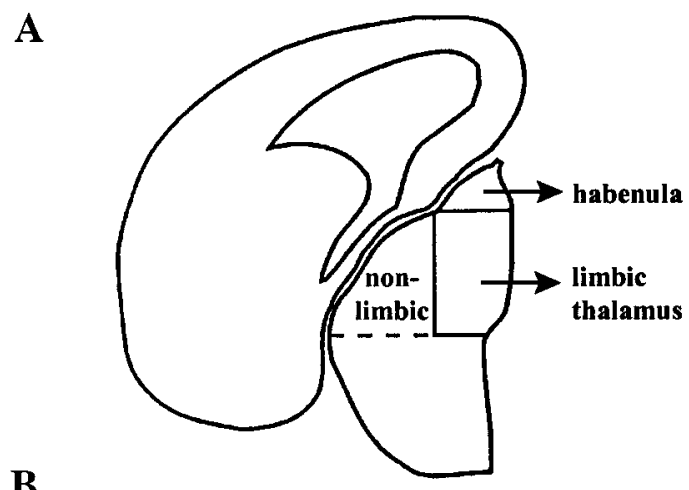

B
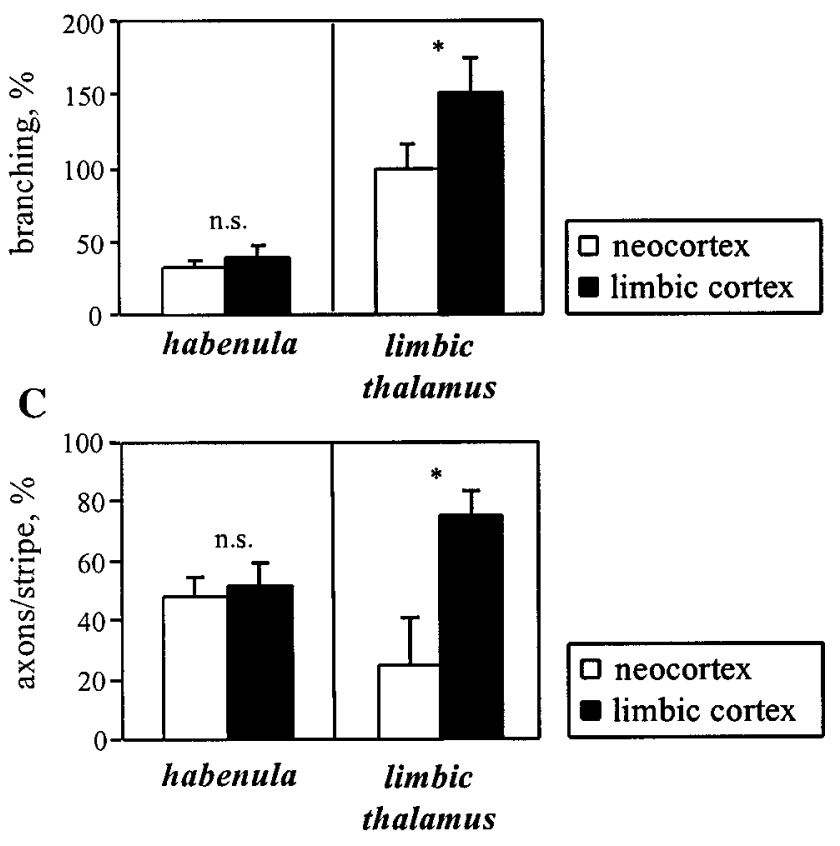

Figure 5. A, Schematic diagram of coronal sections through E19 diencephalon, illustrating the dissection of the habenular complex from the limbic dorsal thalamus. $B$, Quantitative analysis for branch formation on membrane substrates from limbic cortex and neocortex. Data normalized to limbic thalamic axons on neocortical membranes. $C$, Quantitative analysis of axonal growth in stripe assay. n.s., Nonsignificant; *significantly different with $p<0.05$.

emission of collaterals on their target membranes compare to neocortical membranes [Fig. 2, $E, n=90$ thalamic axons (neocortex), $n=90$ (limbic cortex); $n s ; F, n=36$ cortical axons (neocortex), $n=43$ (limbic cortex); NS]. On the other hand, neocortical fibers were not affected by the antibody treatment and continued to branch more often on their target membranes [Fig. $2 F, n=58$ (neocortex), $n=58$ (limbic cortex); $p<0.05$ ]. Antibody treatment abolished the preference of limbic thalamic axons for limbic cortical stripes (Fig. 6), resulting in an equivalent distribution of the fibers on both membrane species (Fig. $4 E, n=$ 47 pairs of stripes; NS). These data indicate that the expression of LAMP in limbic cortical membranes is sufficient to mediate choices for appropriate limbic cortical and thalamic fibers.

The presence of anti-LAMP changed the branching characteristics of nonlimbic thalamic fibers, resulting in significantly more branching on their nontarget membranes than on target membranes [Fig. $2 E, n=39$ (neocortex), $n=38$ (limbic cortex); $p<$ $0.05]$. However, nonlimbic thalamic axons, in the presence of the antibody, continued to grow preferentially on their target neocortical membranes in the stripe assay (Fig. $4 E, n=33$ pairs of stripes; $p<0.05)$. These results suggest that although LAMP alone can affect the growth of nonlimbic thalamic fibers (Fig. 4A), the differential expression of LAMP in the cortex is not sufficient for nonlimbic thalamic fibers to discriminate their appropriate substrate.

\section{Axonal interactions in guidance of limbic fibers}

The expression of LAMP on developing axons and growth cones (Horton et al., 1988; Zacco et al., 1990) is consistent with the ability of LAMP to regulate axo-axonal interactions during development of limbic projections. To test this possibility, the behavior of limbic cortical and thalamic axons, after contact, was analyzed by time-lapse video microscopy. Previous studies reported that contacts between two growth cones or a growth cone and an axon can lead to three distinctive behaviors: fasciculation, retraction, and crossing (Bolz et al., 1995). Figure $7 A$ illustrates a growth cone from a limbic cortical neuron fasciculating along a limbic thalamic fiber. Identical fasciculations occurred in the situation in which a growth cone of a limbic thalamic neuron encountered a limbic cortical fiber. Quantitative analysis indicated that $68 \%$ of the interactions between limbic cortical and limbic thalamic axons lead to a fasciculation, whereas only $5 \%$ of contacts ended by a crossing, and $27 \%$ ended by a retraction $(n=$ 40 contacts recorded). To determine the influence of the limbic phenotype on axo-axonal interactions, contacts between two cortical axons extending respectively from a limbic and a neocortical explant were studied. As seen in Figure $7 B$, these two axonal types usually retracted from each other. In $80 \%$ of interactions observed, cortical axons retracted, whereas in only $20 \%$ of the cases, contacts between limbic cortical and neocortical fibers led to a fasciculation $(n=15)$.

In the presence of anti-LAMP the number of fasciculations between limbic fibers from cortical and thalamic explants decreased from 68 to $33 \%$, whereas retractions became predominant and increased to $62 \%(n=21)$. In contrast, when antiLAMP was added to the cultures of limbic cortical and neocortical fibers, they continued to retract from each other $(n=$ 6). Our data suggest that fasciculation between limbic thalamic and limbic cortical axons is LAMP-sensitive, whereas retraction between limbic cortical fibers and neocortical fibers occurs independently of LAMP.

\section{DISCUSSION}

In the adult mammalian brain, thalamocortical projections are organized such that specific thalamic nuclei project to specific regions of the cerebral cortex. Axonal tracing studies demonstrate that the targeting of thalamocortical axons to their appropriate cortical area during development is very precise (Wise and Jones, 1978; Crandall and Caviness, 1984; De Carlos and O'Leary, 1992; Erzurumlu and Jhaveri, 1992; Agmon et al., 1995). Additionally, the absence of target subplate neurons fails to evoke nonspecific thalamic ingrowth into neighboring cortical areas (Ghosh and Shatz, 1993). The molecular mechanisms that control the specificity of thalamocortical projections are unknown. Results of in vitro experiments described here provide evidence for the role of differentially expressed membrane-associated signals in guiding specific types of thalamic fibers and regulating their branching.

\section{Specification of thalamocortical projections in vitro}

Previous studies have shown that co-cultured thalamic and cortical explants establish layer-specific interconnections (Bolz et al., 
Figure 6. Phase-contrast photomicrographs of explants harvested from limbic thalamus on alternating stripes of membranes from limbic cortex (limb) and neocortex (neo) under control conditions $(A)$ and after addition of antiLAMP $(C) . B, D$, Higher-magnification views of outlined areas in $A$ and $C$, respectively. The antibody treatment reduces the preference of limbic thalamic axons for their target membranes. In each case, membranes from limbic cortex are identified with fluorescent beads and labeled limb, as shown on the right. Scale bars, $100 \mu \mathrm{m}$.

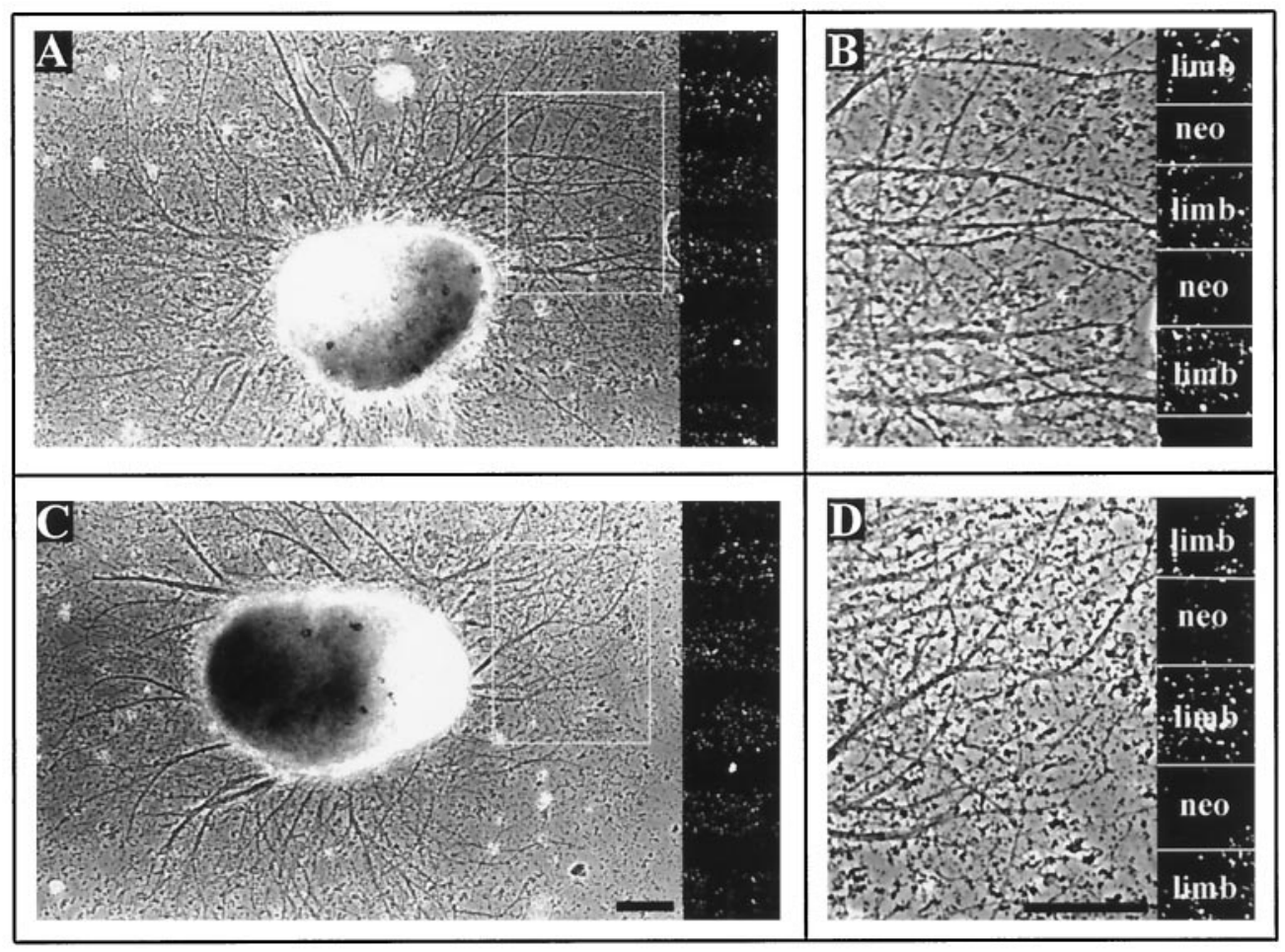

1992; Yamamoto et al., 1992). However, in vitro studies also indicated that thalamocortical connections lack areal specificity; explants from lateral (visual) thalamus project into slices of visual cortex as well as into slices from somatosensory cortex, and axons from explants that include somatosensory thalamus (ventrobasal nucleus) innervate both somatosensory and visual cortex (Molnár and Blakemore, 1991; Yamamoto et al., 1992). We also found that limbic and nonlimbic thalamic explants innervate both neocortical and limbic cortical slices (data not shown) and that they extend the same number of axons on a homogenous membrane carpet prepared from either limbic cortex or neocortex. Based on these in vitro findings, it has been suggested that a trophic signal attracts thalamic fibers to the cortex but provides no positional information (Blakemore and Molnár, 1990; Molnár and Blakemore, 1995). Other studies, however, demonstrate that thalamocortical innervation is controlled by membrane-bound, growthpromoting molecules, which become upregulated in the developing cortical plate (Götz et al., 1992; Hübener et al., 1995; Tuttle et al., 1995). Because these signals are expressed throughout the cerebral cortex, they might allow the ingrowth of thalamic axons into slices from different cortical regions.

Co-culture experiments, however, do not exclude the possibility that molecular cues intrinsic to the cortex provide positional information for thalamocortical axons. First, such experiments do not represent a quantitative assay for thalamic fiber growth. Second, they do not provide alternative targets for thalamic axons. Third, thalamic explants may include neurons from nonspecific thalamic nuclei that do not show growth preference in vivo. The results presented here indicate that limbic and nonlimbic axons, when given a choice between membrane substrates prepared from limbic cortex and neocortex, grow preferentially on membranes from their cortical target region. Moreover, our quantitative growth assays revealed that thalamic axons exhibit more branches on membranes from cortical target regions than on membranes from nontarget regions. Thus, these in vitro findings indicate that membrane-associated molecules allow thalamic axons to distinguish between neocortical and limbic cortical regions and are consistent with anatomical evidence from in vivo analysis.

\section{LAMP and the specification of limbic thalamocortical circuits}

The early and restricted expression of LAMP in cortical and subcortical limbic regions, which are highly interconnected by specific neuronal projections, led to the proposal that this molecule serves as a recognition signal for the formation of limbic circuits (Levitt, 1984; Pimenta et al., 1995; Levitt et al., 1997). Consistent with this idea, transplantation studies using cortical explants from fetuses at different ages showed that the expression of the LAMP phenotype is predictive of the class of thalamic (Barbe and Levitt, 1992) and cortico-cortical inputs (Barbe and Levitt, 1995) established by the graft tissue. Recent studies indicated that LAMP facilitates adhesion and neurite outgrowth of isolated neurons from limbic cortex and hippocampus but not of nonlimbic neurons from olfactory bulb and neocortex (Pimenta et al., 1995; Zhukareva and Levitt, 1995). Because LAMP is capable of mediating aggregation in a cell-free system (Zhukareva and Levitt, 1995), it has the characteristics of a homophilic cell adhesion molecule. Consistent with these results, we found a branch-promoting and an attractive guidance effect of LAMP only for limbic thalamic and cortical axons.

How might the effects observed with recombinant LAMP in a controlled environment relate to the action of native LAMP in vivo? In the stripe assay with $\mathrm{CHO}$ cell membranes, limbic cortical axons grew preferentially on membranes from rLAMPtransfected cells. However, in our experiments with alternating membranes prepared from limbic cortex and neocortex, the same fiber population failed to exhibit a preference. Thus, either the amount of LAMP in native membrane preparations is not suffi- 

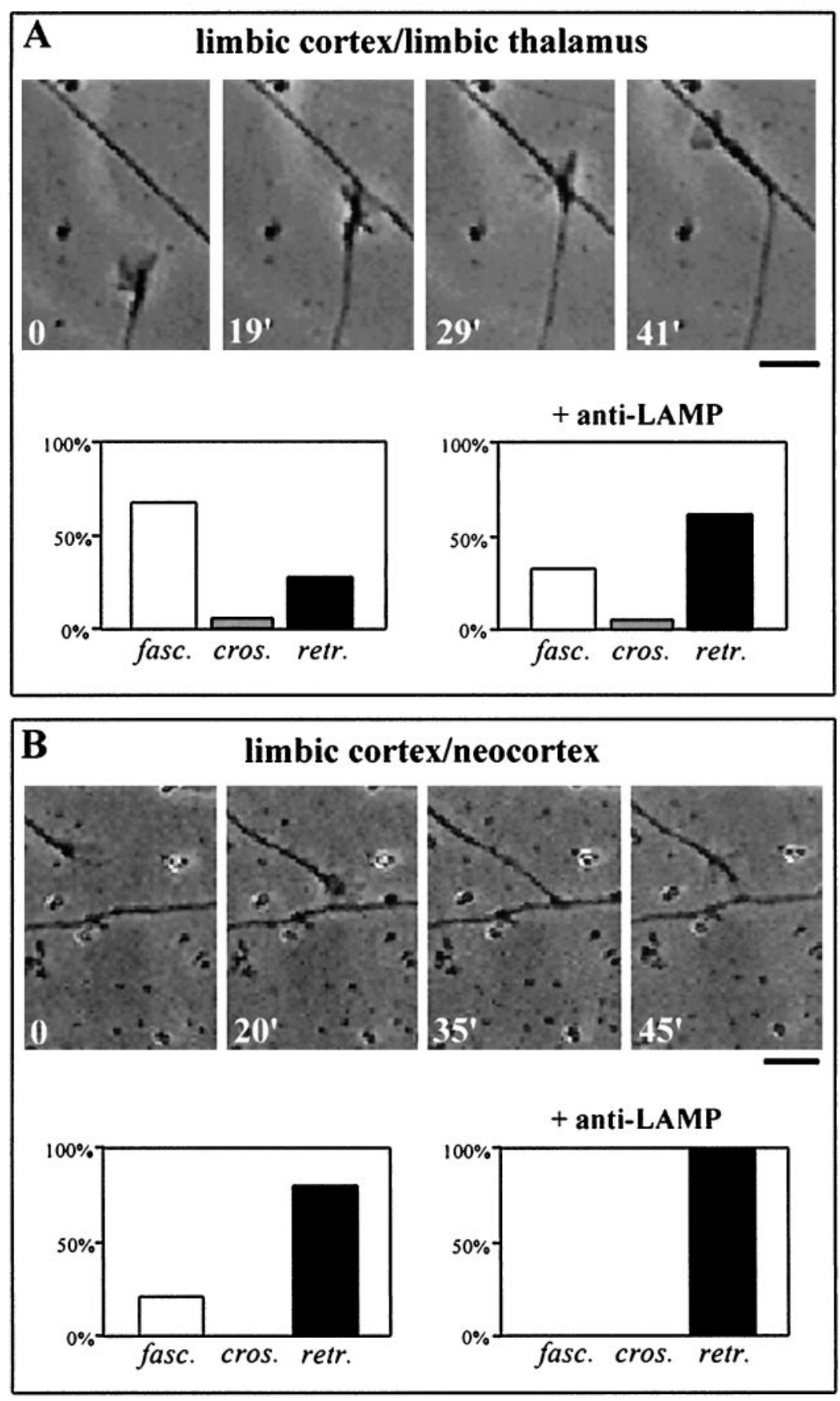

Figure 7. Quantitative analysis of axo-axonal interactions between limbic cortical and limbic thalamic axons $(A)$ and limbic cortical and neocortical axons (B) under control conditions and in the presence of anti-LAMP. Bars indicate for each axonal combination the percentage of fasciculation (open bars), crossing (gray bars), and retraction (black bars) observed. Sequences of video micrographs illustrate representative axo-axonal interactions: $A$, a limbic cortical growth cone fasciculates along a limbic thalamic fiber; $B$, a neocortical growth cone retracts after contact with a limbic cortical axon. Time (in minutes) is indicated on each panel. Scale bar, $10 \mu \mathrm{m}$. cient to steer the growth cones of these axons, or other molecules present in membrane preparations override or interfere with the capability of LAMP to guide limbic cortical axons in this assay system. In contrast, limbic thalamic axons were effectively guided in the stripe assay with native cortical membranes. The preference of limbic thalamic axons for the membrane stripes from limbic cortex was completely abolished by antibodies directed against LAMP, indicating that LAMP is capable of controlling the guidance of limbic thalamic axons in a complex molecular environment. Likewise, the ability of antibodies to block the increase in axonal branching observed on limbic cortical membranes suggests that this cellular response can be regulated by LAMP under in vivo conditions.
There is substantial evidence that cell adhesion molecules on axonal surfaces can promote fiber fasciculation by either homophilic or heterophilic interactions (for review, see Reichardt and Tomaselli, 1991; Goodman, 1996). LAMP is transiently expressed on developing limbic axons along fiber pathways in vivo, and it mediates homophilic binding in vitro. Therefore, it was proposed that LAMP is involved in axon fasciculation (Pimenta et al., 1995). The present time-lapse imaging experiments provide the first direct evidence for the specificity of LAMP in mediating fasciculation between limbic axons. The modulation of axonal branching, guidance, and fasciculation by LAMP suggests that it can actively contribute to the specification of limbic circuits. 


\section{Alternative functions of LAMP in the neocortical system}

The response of limbic axons to LAMP, described in the previous section, can in principle be explained by a homophilic binding mechanism that promotes axonal growth and branching. However, we also observed that under some conditions nonlimbic thalamic neurons, which do not express LAMP, are sensitive to this molecule. Thus, LAMP may also function as a heterophilic ligand for cell surface molecules on distinct populations of nonlimbic axons. The heterophilic interactions shown in our assay system reveal a novel function of LAMP: in contrast to the growth-promoting effects observed with limbic axons, LAMP reduced axonal growth and branching and acted as a repulsive guidance signal for nonlimbic thalamic axons. Such opposing functions of an axonal guidance signal have also been described for members of the netrin and ephrin gene family (Tamada et al., 1995; Shirasaki et al., 1996; Bolz and Castellani, 1997; Castellani et al., 1998).

The recent identification of the coding sequences of different axon guidance proteins revealed in several cases a striking phylogenetic conservation of their molecular structure and their cellular function. For example, netrins in the mouse have $\sim 50 \%$ sequence homology with the UNC-6 protein in the nematode, and both netrins and UNC-6 are secreted molecules that attract circumferential growing axons (Ishii et al., 1992; Serafini et al., 1996). The present findings suggest that a guidance cue preferentially expressed in the limbic system, a phylogenetically old part of the vertebrate brain, may also be used as a guidance signal in the developing neocortical system, the evolutionary youngest part of the mammalian brain, but interpreted in an opposing way. Thus, whereas LAMP may serve as a positive cue for thalamic axons destined to project to the limbic cortex, it could serve as a negative cue for thalamic axons destined to innervate the neocortex. One implication of these findings is that defects in the regulation or expression of the lamp gene during development not only would affect limbic circuits but would also have repercussions for the assembly of neocortical circuits. The production of a lamp-null mouse may address these issues.

\section{Are there neocortical system-associated membrane proteins?}

Although LAMP exerts an inhibitory effect on nonlimbic thalamic axons via heterophilic interactions, this effect alone does not explain why these fibers exhibit the ability to distinguish between limbic and nonlimbic cortical regions. In the stripe assay, antibody treatment of native cortical membranes did not interfere with the preference of nonlimbic axons for neocortical membranes, whereas rLAMP in CHO cell membranes exerts a repellent effect. One possible explanation is that native limbic membranes contain other repulsive molecules for nonlimbic axons, for example, members of the Eph family (Zhou et al., 1994). Alternatively, signals selectively expressed in the neocortex could contribute to the targeting of thalamic axons. Although there is now increasing evidence for an early molecular specification of cortical areas (Arimatsu et al., 1992; Cohen-Tannoudji et al., 1994; Paysan et al., 1994; Suzuki et al., 1997) (for review, see Levitt et al., 1997), there is still very little known about specific guidance factors in neocortical regions. Previous studies demonstrated that neocortical fibers have a strong tendency to fasciculate with each other (Bolz et al., 1995). The present time-lapse recordings demonstrated that limbic cortical axons retract after contact with neocortical axons. Together, these results provided evidence for unique attractive and repulsive molecules expressed by neocortical and limbic cortical neurons that influence growth cone behavior of the appropriate thalamic axon population.

\section{REFERENCES}

Agmon A, Yang LT, Jones EG, O’Dowd DK (1995) Topological precision in the thalamic projection to neonatal mouse barrel cortex. J Neurosci 15:549-561.

Altman J, Bayer SA (1979) Development of the diencephalon in the rat. VI. Re-evaluation of the embryonic development of the thalamus on the basis of thymidine-radiographic datings. J Comp Neurol 188:501-524.

Arimatsu Y, Miyamoto M, Nihonmatsu I, Hirata K, Uratani Y, Hatanaka Y, Takiguchi-Hayashi K (1992) Early regional specification for a molecular neuronal phenotype in the rat neocortex. Proc Natl Acad Sci USA 89:8879-8883.

Barbe MF, Levitt P (1992) Attraction of specific thalamic input by cerebral grafts depends on the molecular identity of the implant. Proc Natl Acad Sci USA 89:3706-3710.

Barbe MF, Levitt P (1995) Age-dependent specification of the corticocortical connections of cerebral grafts. J Neurosci 15:1819-1834.

Bayer SA, Altman J (1991) Neocortical development. New York: Raven.

Blakemore C, Molnár Z (1990) Factors involved in the establishment of specific interconnections between thalamus and cerebral cortex. Cold Spring Harb Symp Quant Biol 55:491-504.

Bolz J, Castellani V (1997) How do wiring molecules specify cortical connections? Cell Tissue Res 290:307-314.

Bolz J, Novak N, Staiger V (1992) Formation of specific afferent connections in organotypic slice cultures from rat visual cortex co-cultured with lateral geniculate nucleus. J Neurosci 12:3054-3070.

Bolz J, Kossel A, Bagnard D (1995) The specificity of interactions between cortex and thalamus. In: Development of the cerebral cortex (Blakemore C, ed), pp 173-191. New York: Wiley.

Bradford MM (1976) A rapid and sensitive method for the quantitation of microgram quantities of protein utilizing the principle of protein dye-binding. Anal Biochem 72:248-254.

Bulfone A, Smiga SM, Shimamura K, Peterson A, Puelles L, Rubenstein JLR (1995) T-brain-1: a homolog of Brachyury whose expression defines molecularly distinct domains within the cerebral cortex. Neuron 15:63-78.

Castellani V, Yue Y, Gao PP, Zhou R, Bolz J (1998) Dual action of a ligand for Eph tyrosine kinases on specific populations of axons during development of cortical circuits. J Neurosci 18:4663-4672.

Cohen-Tannoudji M, Babinet C, Wassef M (1994) Early determination of a mouse somatosensory cortex marker. Nature 368:460-463.

Crandall JE, Caviness VS (1984) Thalamocortical connections in newborn mice. J Comp Neurol 228:542-556.

De Carlos JA, O'Leary DDM (1992) Growth and targeting of subplate axons and establishment of major cortical pathways. J Neurosci 12:1194-1211.

Deacon TW, Eichenbaum H, Rosenberg P, Eckmann KW (1983) Afferent connections of the perirhinal cortex in the rat. J Comp Neurol 220:168-190.

Erzurumlu RS, Jhaveri S (1992) Emergence of connectivity in the embryonic rat parietal cortex. Cereb Cortex 2:336-352.

Ghosh A, Shatz CJ (1993) A role for subplate neurons in the patterning of connections from thalamus to neocortex. Development 117: 1031-1047.

Goodman CS (1996) Mechanisms and molecules that control growth cone guidance. Annu Rev Neurosci 19:341-377.

Götz M, Novak N, Bastmeyer M, Bolz J (1992) Membrane bound molecules in rat cerebral cortex regulate thalamic innervation. Development 116:507-519.

Hatanaka Y, Uratani Y, Takiguchi-Hayashi K, Omori A, Sato K, Miyamoto M, Arimatsu Y (1994) Intracortical regionality represented by specific transcription for a novel protein, latexin. Eur J Neurosci 6:973-982.

Henke-Fahle S, Mann F, Götz M, Wild K, Bolz J (1996) A dual action of a carbohydrate epitope on afferent and efferent axons in cortical development. J Neurosci 16:4195-4206.

Horton HL, Levitt P (1988) A unique membrane protein is expressed on early developing limbic systems axons and cortical targets. J Neurosci 8:4653-4661.

Hübener M, Götz M, Klostermann S, Bolz J (1995) Guidance of 
thalamocortical axons by growth-promoting molecules in developing rat cerebral cortex. Eur J Neurosci 7:1963-1972.

Ishii N, Wadsworth WG, Stern BD, Culotti JG, Hedgecock EM (1992)

UNC-6, a laminin-related protein, guides cell and pioneer axon migrations in C. elegans. Neuron 9:873-881.

Keller F, Rimvall K, Barbe MF, Levitt P (1989) A membrane glycoprotein associated with the limbic system mediates the formation of the septo-hippocampal pathway in vitro. Neuron 3:551-561.

Krettek JE, Price JL (1977) The cortical projections of the mediodorsal nucleus and adjacent thalamic nuclei in the rat. J Comp Neurol 171:157-192.

Levitt P (1984) A monoclonal antibody to limbic system neurons. Science 223:299-301.

Levitt P, Barbe MF, Eagleson KL (1997) Patterning and specification of the cerebral cortex. Annu Rev Neurosci 20:1-24.

McAllister JP, Das GD (1977) Neurogenesis in the epithalamus, dorsal thalamus and ventral thalamus of the rat: an autoradiographic and cytological study. J Comp Neurol 172:647-686.

Miller MW (1988) Development of projection and local circuit neurons in neocortex. In: Cerebral cortex, Vol 7, Development and maturation of cerebral cortex (Peters A, Jones EG, eds), pp 133-175. New York: Plenum.

Molnár Z, Blakemore C (1991) Lack of regional specificity for connections formed between thalamus and cortex in coculture. Nature 351:475-477.

Molnár Z, Blakemore C (1995) How do thalamic axons find their way to the cortex? Trends Neurosci 18:389-397.

Paxinos G, Watson C (1982) The rat brain in stereotaxis coordinates. Sydney, Australia: Academic.

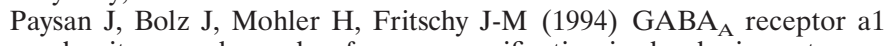
subunit, an early marker for area specification in developing rat cerebral cortex. J Comp Neurol 350:133-149.

Pimenta A, Zhukareva V, Barbe MF, Reinoso B, Grimley C, Henzel W, Fischer I, Levitt P (1995) The limbic system-associated membrane protein is an Ig superfamily member that mediates selective neuronal growth and targeting. Neuron 15:287-297.

Pimenta AF, Reinoso BS, Levitt P (1996) Expression of the mRNAs encoding the limbic system-associated membrane protein (LAMP). 2. Fetal rat brain. J Comp Neurol 375:289-302.

Reichardt LF, Tomaselli KJ (1991) Extracellular matrix molecules and their receptors: functions in neural development. Annu Rev Neurosci 14:531-570.

Robertson RT, Kaitz SS (1981) Thalamic connections with limbic cortex. I. Thalamocortical projections. J Comp Neurol 195:501-525.
Serafini T, Colamarino SA, Leonardo ED, Wang H, Beddington R, Skarnes WC, Tessier-Lavigne M (1996) Netrin-1 is required for commissural axon guidance in the developing vertebrate nervous system. Cell 87:1001-1014.

Shirasaki R, Mirzayan C, Tessier-Lavigne M, Murakami F (1996) Guidance of circumferentially growing axons by netrin-dependent and -independent floor plate chemotropism in the vertebrate brain. Neuron 17:1079-1088.

Suzuki SC, Inoue T, Kimura Y, Tanaka T, Takeichi M (1997) Neuronal circuits are subdivided by differential expression of type-II classic cadherins in postnatal mouse brains. Mol Cell Neurosci 9:433-447.

Tamada A, Shirasaki R, Murakami F (1995) Floor plate chemoattracts crossed axons and chemorepels uncrossed axons in the vertebrate brain. Neuron 14:1083-1093.

Tuttle R, Schlaggar BL, Braisted JE, O’Leary DDM (1995) Maturationdependent upregulation of growth-promoting molecules in developing cortical plate controls thalamic and cortical neurite growth. J Neurosci 15:3039-3052.

Walter J, Kern-Veits B, Huf J, Stolze B, Bonhoeffer F (1987) Recognition of position-specific properties of tectal cell membranes by retinal axons in vitro. Development 101:685-696.

Wise SP, Jones EG (1978) Developmental studies of thalamocortical and commissural connections in the rat somatic sensory cortex. J Comp Neurol 175:187-208.

Wizenmann A, Thies E, Klostermann S, Bonhoeffer F, Bähr M (1993) Appearance of target-specific guidance information for regenerating axons after CNS lesions. Neuron 11:975-983.

Yamamoto N, Yamada K, Kurotani T, Toyama K (1992) Laminar specificity of extrinsic cortical connections studied in coculture preparations. Neuron 9:217-228.

Zacco A, Cooper V, Highland-Fisher S, Chantler PD, Horton HL, Levitt P (1990) Isolation, biochemical characterization and ultrastructural localization of the limbic system associated membrane protein (LAMP), a protein expressed on neurons comprising functional neural circuits. J Neurosci 10:73-90.

Zhou R, Copeland TD, Kromer LF, Schulz NT (1994) Isolation and characterization of Bsk, a growth factor receptor-like kinase associated with the limbic system. J Neurosci Res 37:129-143.

Zhukareva V, Levitt P (1995) The limbic system-associated membrane protein (LAMP) selectively mediates interactions with specific central neuron populations. Development 121:1161-1172. 